\title{
GUTACHTERLISTE 2004
}

Die Redaktion der PVS dankt allen Gutachterinnen und Gutachtern, die bei der Auswahl der Beiträge für den Jahrgang 2004 behilflich waren.

\begin{tabular}{|c|c|c|}
\hline Ulrich von Alemann & Cord Jakobeit & Christoph Schaltegger \\
\hline Ralf Altenhof & Otmar Jung & Fritz W. Scharpf \\
\hline Kai Arzheimer & André Kaiser & Stefan Seidendorf \\
\hline Michael Becker & Norbert Kersting & Frank Schimmelfennig \\
\hline Joachim Behnke & Paul Kevenhörster & Josef Schmid \\
\hline Arthur Benz & Bernhard Kittel & Johannes Schmidt \\
\hline Christoph Bieber & Markus Klein & Hermann Schmitt \\
\hline Tanja A. Börzel & Ralf Kleinfeld & Rüdiger Schmitt-Beck \\
\hline Jörg Bogumil & Heinz Kleger & Gerald Schneider \\
\hline Bernhard Boll & Thomas König & Astrid Schütz \\
\hline Rainer Bovermann & Henk van der Kolk & Winfried Schulz \\
\hline Tanja Brühl & Peter Koller & Gunnar Folke Schuppert \\
\hline Hubertus Buchstein & Sascha-Ilko Kowalczuk & Walter Schweidler \\
\hline Horst Dähn & Sabine Kropp & Susumu Shikano \\
\hline Claudia Derichs & Daniel Kübler & Nico A. Siegel \\
\hline Heribert Dieter & Steffen M. Kühnel & Georg Simonis \\
\hline Herbert Döring & Volker Kunz & Barbara Steininger \\
\hline Walter Euchner & Stephan Leibfried & Peter Steinbach \\
\hline Dieter Fuchs & Roland Lhotta & Roland Sturm \\
\hline Thomas Gehring & Susanne Lütz & Ingo Take \\
\hline Manuela Glaab ${ }^{\circ}$ & Jochen Mayerl & Helmut Thome \\
\hline Karsten Grabow & Heiner Meulemann & Paul W. Thurner \\
\hline Edgar Grande & Ursula Münch & Hans Werner Tobler \\
\hline Michael Th. Greven & Oskar Niedermayer & Annette Elisabeth Töller \\
\hline Dieter Grunow & Peter Nitschke & Adrian Vatter \\
\hline Thomas Gschwend & Andreas Nölke & Angelika Vetter \\
\hline Sabine Häder & Claus Offe & Uwe Wagschal \\
\hline Sibylle Hardmeier & Werner J. Patzelt & Christian Welzel \\
\hline Horst Alfred Heinrich & Ingo Peters & Raymund Werle \\
\hline Wolfgang Heinz & Jürgen Plöhn & Bernhard Weßels \\
\hline Everhard Holtmann & Thomas Plümper & Wolfgang Wessels \\
\hline Katharina Holzinger & Walter Reese-Schäfer & Bettina Westle \\
\hline Rudolf Hrbek & Wolfgang Renzsch & Thomas von Winter \\
\hline Simon Hug & Emanuel Richter & Bernhard Zangl \\
\hline Markus Jachtenfuchs & Sigrid Roßteutscher & Reimut Zohlnhöfer \\
\hline Detlef Jahn & Richard Saage & \\
\hline
\end{tabular}

